\title{
Real-Time High-Rate GNSS Displacements: Performance Demonstration During the 2019 Ridgecrest, CA Earthquakes
}

\author{
Diego Melgar ${ }^{1 \dagger}$, Timothy I. Melbourne ${ }^{2}$, Brendan W. Crowell ${ }^{3}$, Jianghui Geng ${ }^{4}$, Walter \\ Szeliga ${ }^{2}$, Craig Scrivner ${ }^{2}$, Marcelo Santillan ${ }^{2}$, and Dara E. Goldberg ${ }^{1}$ \\ ${ }^{1}$ Department of Earth Sciences, University of Oregon, Eugene, OR, USA \\ ${ }^{2}$ Pacific Northwest Geodetic Array, Department of Geological Sciences, Central Washington \\ University, Ellensburg, WA, USA \\ ${ }^{3}$ Department of Earth and Space Sciences, University of Washington Seattle, WA, USA \\ ${ }^{4}$ GNSS Center, University of Wuhan, Wuhan, China
}

+Corresponding author: dmelgarm@uoregon.edu

This is a non-peer reviewed pre-print submitted to EarthArXiv. This paper has been submitted to Seismological Research Letters for review.

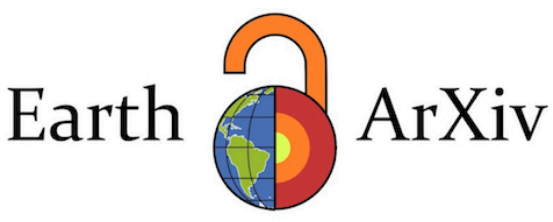




\begin{abstract}
Traditional real-time seismology has relied on inertial sensors to characterize ground motions and earthquake sources, particularly for hazards applications such as warning systems. In the past decade, a revolution in high-rate, real-time Global Navigation Satellite System (GNSS) displacement have provided a new source of data to augment traditional measurement devices. The Ridgecrest, California earthquake sequence in 2019 provided one of the most complete recordings of real-time GNSS displacements to date, helping to aid in an initial source characterization over the first few days. In this manuscript, we analyze and make available the archived real-time displacement streams and compare their performance to post-processed results, which we also provide. We find good agreement for all stations showing a noticeable signal. This demonstrates that simple modeling in real-time, such as peak ground displacement scaling, would be practically identical to post-processed results. Similarly, we find good agreement across the full spectral range, from the coseismic offsets $(\sim 0 \mathrm{~Hz})$ to the Nyquist frequency. We also find low latency between the measurement acquisition at the field site and the position calculation at the datacenter. In aggregate, the performance during the Ridgecrest earthquakes is strong evidence of the viability and usefulness of real-time GNSS as a monitoring tool.
\end{abstract}

\title{
Introduction
}

Global Navigation Satellite Systems (GNSS) comprise constellations of navigation satellites such as the Global Positioning System (GPS). GNSS satellites broadcast microwave signals that are recorded by land-based antennas and receivers and can be used to obtain the position of the antenna. If the antenna moves, as it would during an earthquake, the position changes can be calculated in real-time to obtain displacement seismograms. GNSS displacement waveforms have accuracy at the cm-level and so are most useful as a ground motion recording instrument during moderate to large events and at local to regional distances (e.g. Nikolaidis, 2001, Larson et al., 2003; Bock et al., 2011). They complement strong motion recordings because they are a non-inertial measurement system and do not suffer from baseline offsets traditionally found in accelerograms (e.g. Boore \& Bommer, 2005). Baseline offsets in accelerograms make unambiguous integration to displacement difficult and effectively limit the usefulness of the resulting waveforms, typically to periods shorter than $\sim 10$ s (e.g. Melgar et al., 2013). In contrast, high-rate (HR) GNSS recordings, usually sampled at $1-10 \mathrm{~Hz}$, provide reliable strong motion displacement measurements down to $\mathrm{OHz}$ (the static offset).

This ability of HR-GNSS displacement recordings to supplement strong motion accelerations to capture the full complexity of ground motion has long been recognized; reviews of the progress in this regard can be found in Bock \& Melgar (2016) and Larson (2019). Databases of postprocessed recordings have been made available for earthquakes in the M6-M9 range (Ruhl et al., 2018). It has been demonstrated that RT-GNSS can be used to obtain rapid magnitudes (e.g. Crowell et al., 2013, Melgar et al., 2015) often before rupture is complete. It can also be used to obtain higher-order earthquake source products such as moment tensors (Melgar et al., 2012, O'Toole et al., 2012, Riquelme et al., 2015) and distributed slip (e.g. Crowell et al., 2012; Wright 
et al., 2012,). As a result, RT-GNSS is being incorporated into both earthquake and tsunami early warning systems (Crowell et al., 2016, Kawamoto et al., 2016, Murray et al., 2018).

However, real-time positioning is challenging. Unlike inertial seismic sensors, which directly record ground motion, GNSS relies on measuring the time-of-flight of the microwave signals from the antenna to the broadcasting satellite to obtain the "pseudorange". Since many satellites are visible at any point in time (typically more than 6 ) the pseudoranges can be combined to solve for the position of the antenna at any point in time. Measurements of the phase with which the microwave signals arrive are further used to refine these solutions to the cm-level of accuracy. For point positioning, in which a GNSS receiver is positioned within a global reference frame rather than relative to nearby stations that are held fixed (Zumberge et al., 1997), positioning depends on knowledge of variables external to the ground motion produced by the earthquake. Two important factors are: a precise knowledge of the satellite clocks, needed to determine when the microwave signal is broadcast by the satellite; and the satellite orbits, necessary to determine the position of the satellites in the sky. Due to myriad factors such as solar wind pressure, relativity, and others, both clocks and orbits drift with time. In post-processing, "final" clocks and orbits, determined with about 2 weeks latency by the International GNSS Service (IGS), can be used to convert the raw GNSS phase and range recordings to the displacement waveforms that have now become commonplace. In real-time, however, lower accuracy "broadcast" satellite orbits and clocks are insufficiently accurate for point-positioning and must therefore be continually updated with corrections derived from continuous regional or global analyses. Thus the process of positioning any given station in real-time entails streaming in both raw satellite observables made at that station as well as a variety of ancillary products derived from separate analyses. All of this makes HR-GNSS positioning more difficult than using an inertial sensor, and potentially degrades the ultimate precision of estimated displacements.

Real-time positioning has been discussed for almost two decades now (e.g. Nikolaidis et al., 2001) and many countries operate real-time GNSS networks as a result of these efforts. However, most of the real-time components of such networks have been circumscribed to telemetering the raw GNSS observables (pseudorange and phase) in real time from the field to a data center. While a number of real-time positioning codes have been described and made available (e.g. Geng et al. 2013, 2019), all of the previous work that discussed the quality and usefulness of GNSS waveforms for real-time seismology has used raw data collected in real-time, but with positions obtained in a post-processing mode. Few, if any, reports exist of actual operational performance of RT-GNSS during a significant earthquake.

The Geodesy Laboratory at Central Washington University (CWU) has for several years been producing real-time positions with a sample rate of $1 \mathrm{~Hz}$ from stations across the West Coast of the US using CWU's Fastlane positioning system. These data are served to the U.S. Geological Survey for use in the ShakeAlert earthquake early warning system (Murray et al., 2018) and to the National Oceanographic and Atmospheric Administration for a pilot project on tsunami warning (Melbourne et al., 2018). During the 2019 July 4th M6.4 and July 7th M7.1 Ridgecrest, CA, earthquakes (Figure 1) roughly 700 stations from the Network of the Americas (NOTA) operated by UNAVCO, Inc. were being positioned in real-time with Fastlane by CWU. Fastlane 
employs carrier phase-only based positioning rather than pseudorange because phase alone is far less contaminated by multipath error, one of the largest sources of noise in high-rate positioning. Fastlane depends on a highly efficient algorithm for the resolution of carrier phase initial ambiguities, which for most stations can be initially resolved in 20-30 seconds. Here we will compare these truly end-to-end real-time positions against solutions obtained after the earthquakes with final clocks and orbits. We will show that, while compared to the final solutions real-time positions are noisier, they are reliable enough to be useful for real-time characterization of the earthquakes.

\section{Observational Setting: GNSS Recordings}

Figure 1 shows the NOTA permanent GNSS stations that recorded the Ridgecrest sequence. Of these, a subset of 9 were being positioned in real-time by CWU during the earthquake and had displacement signals larger than the background noise. We focus our analysis on this subset, which includes recordings of both earthquakes.

In order to assess the quality of the real-time displacement solutions we compare them against post-processed $1 \mathrm{~Hz}$ solutions obtained using the precise point positioning (PPP) code described by Geng et al. (2019). We used the rapid satellite orbit, phase clock and phase bias products generated by Wuhan University with a latency of 24 hours to enable postprocessed PPP solutions with integer ambiguities resolved. The latest IGS antenna phase center model, code bias model and IERS (International Earth Rotation and Reference Systems Service) solid Earth tide, ocean tidal displacement and pole tide models were applied. We also used global mapping functions to project zenith tropospheric delays onto slant directions with standard meteorological parameters. Examples of the two sets of $1 \mathrm{~Hz}$ waveforms, post-processed and realtime, can be seen in Figure 2. There are evident differences between them, the real-time solutions are noisier. A detailed discussion and analysis of these differences will follow. Here we are also interested in examining any impacts that the sample rate might have. Since the NOTA data is also recorded at $5 \mathrm{~Hz}$, for each of the analyzed stations we produced higher rate $5 \mathrm{~Hz}$ sampled

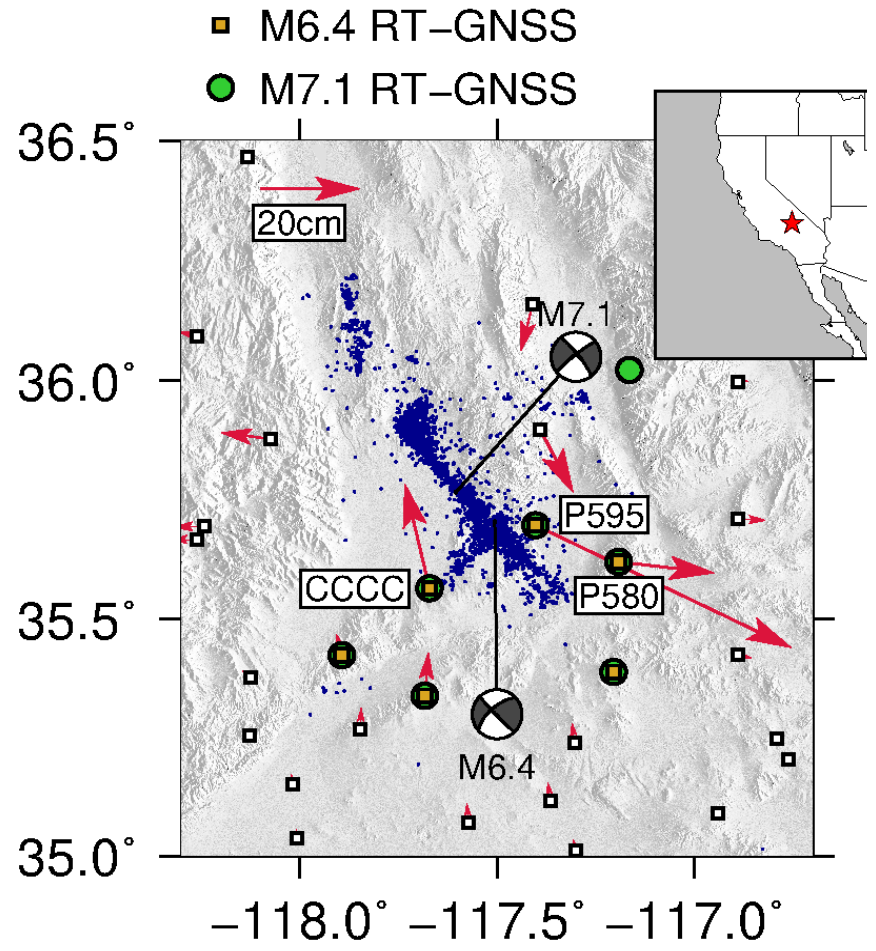

Figure 1. Real-time stations analyzed in this study. White square denote the NOTA permanent sites. The small dots are aftershocks from the first 2 weeks after the July 4th M6.4 earthquake. Arrows are coseismic offsets for the M7.1 earthquake 
positions as well. The $1 \mathrm{~Hz}$ real-time and 1 and $5 \mathrm{~Hz}$ post-processed data is made available in miniSEED format (see Data and Resources)
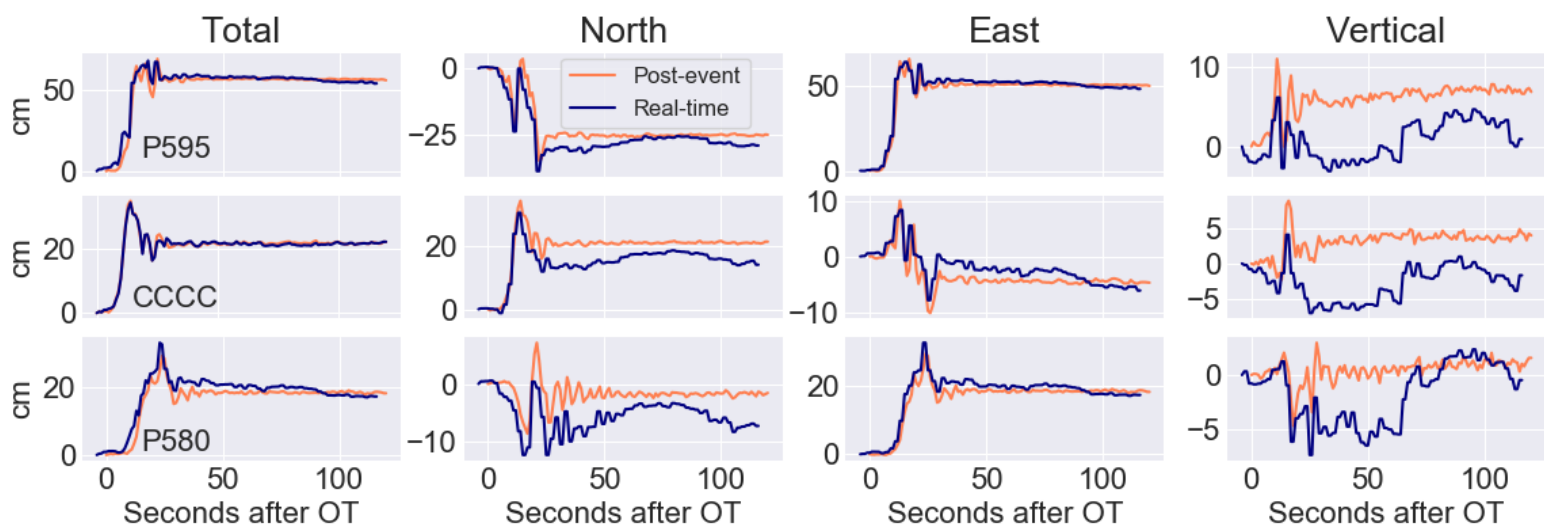

Figure 2. Examples of GNSS waveforms for the M7.1 earthquake. Station locations are in Figure 1.

Finally, we study the impact of the higher noise real-time solutions on the features of the displacement waveforms typically used for warning and hazards applications, namely the peak ground displacement (PGD) and the coseismic, or static offsets. For PGD we can compare directly between real-time and post-processed solutions. For the coseismic offsets we rely on post-event offset estimations from the UNAVCO GAGE Facility for both M6.4 and M7.1 events (Herring et al., 2016). The offsets from the M7.1 earthquake are shown in Figure 1.

\section{Data Quality}

As shown in Figure 2 there are obvious differences between real-time and post-processed data. When the signals are small, as in the vertical components, the differences are easier to spot. This increased noise level in the real-time solutions impacts the frequency domain performance as well. Figure 3 shows an example of the amplitude spectrum for the three components of displacement at station P595 during the M7.1 earthquake. This was calculated using the multitaper method of Prieto et al. (2009). The overall spectra have similar shapes but there are differences across all frequencies. To understand if there is a systematic pattern to this we calculate the individual spectral biases $b_{i k}$ at a station $i$ and at frequency $f_{k}$ as

$$
b_{j k}=\ln \frac{A_{R T}^{i}\left(f_{k}\right)}{A_{P}^{i}\left(f_{k}\right)} \text {, }
$$

Where In is the natural logarithm, $A_{R T}^{i}\left(f_{k}\right)$ is the real-time amplitude spectrum at station $i$ and frequency $f_{k}$, and $A_{P}^{i}\left(f_{k}\right)$ is the amplitude spectrum for the post-processed waveform. Then for all the stations that recorded both the M6.4 and M7.1 earthquakes we calculate the total spectral bias as: 


$$
B\left(f_{k}\right)=\sum_{I=1}^{n} b_{i k}
$$
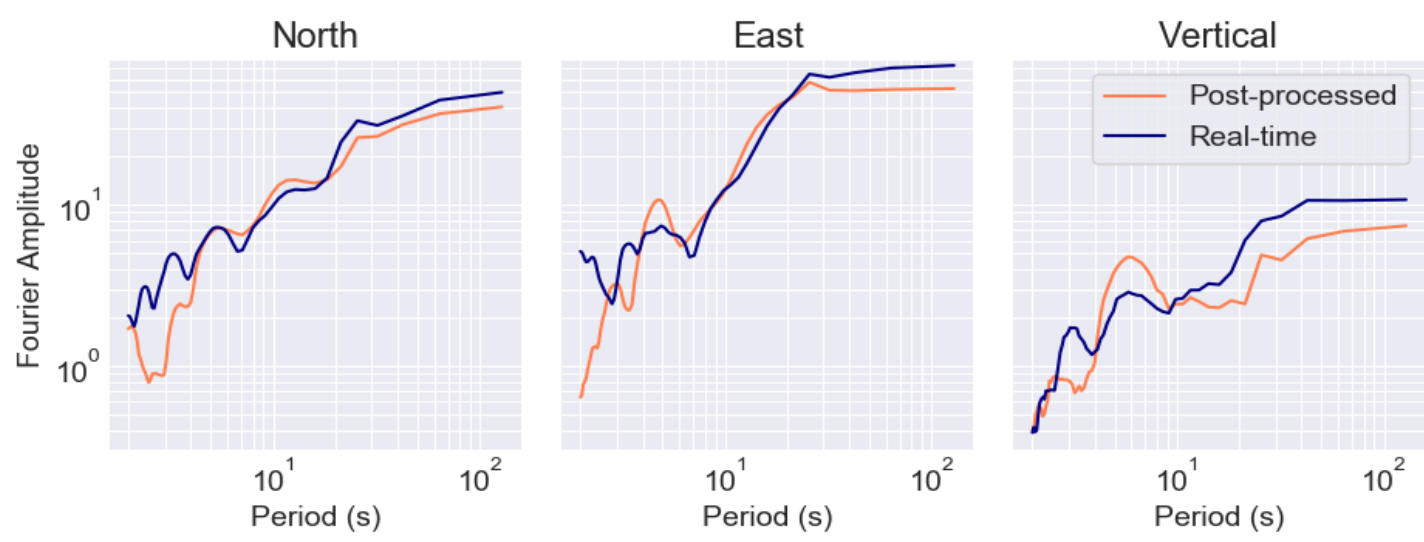

Figure 3. Multitaper estimates of the amplitude spectra for station P595 during the M7.1 earthquake.

where $\mathrm{n}$ is the total number of waveforms. The results are shown in Figure 4. Positive values indicate that the spectral content at a particular frequency is larger in the post-processed data, negative biases that the spectral content is larger in the real-time waveforms. A value close to zero indicates that the spectral content is similar. The results show a "sweet-spot" for these waveforms between $\sim 4-30$ s period where the frequency content is very similar. This is consistent with the periods at which one would expect significant radiation (e.g. Beresnev \& Atkinson, 1997) given the expected source duration for an M6.4 and M7.1 earthquake. At periods shorter and longer than that, $B$ is consistently negative indicating that the noise in the real-time time series introduces a consistent spectral bias.

Given these differences between real-time and post-processed data, it is important to quantify the impacts to the two most common features of the displacement waveforms used for early warning and hazards, the PGD and the coseismic offsets. Figure 5 shows the difference between the real-time and post-processed PGD values. They all fall close to the 1:1 line and the standard deviation of the difference between them is $6.5 \mathrm{~cm}$. Assuming that the post-processed value is the ground truth, then this standard deviation is an empirical estimate of the real-time uncertainty in PGD. If considering a 3-component PGD, there is one significant outlier. If the PGD is

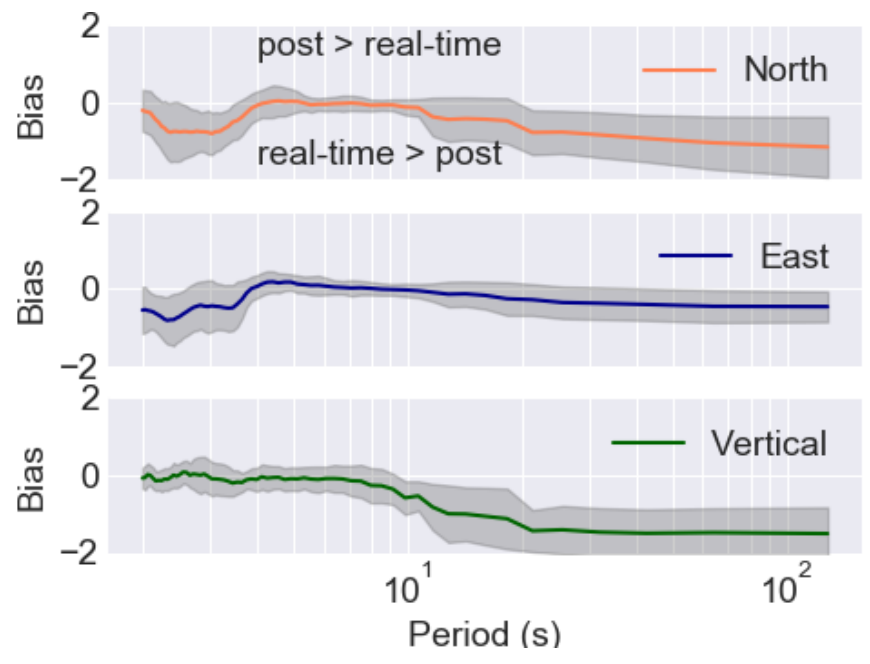

Figure 4. Amplitude spectra bias in nondimensional units between real-time and postprocessed data. The biases are calculated over all stations and for both earthquakes. The shaded region indicates one standard deviation 
calculated only on the horizontal component (Figure 5b) then the standard deviation improves to $4.1 \mathrm{~cm}$. As noted previously by Melgar et al. (2015) for moderate ( M7) magnitude events where GNSS noise is close to the size of the observed signals, considering only the horizontal PGD can lead to more robust measurements. This is supported by what is seen for the Ridgecrest earthquakes. This is not surprising as the vertical component is on average 5 times noisier than the horizontal components (Bock et al., 2011) and the noise level of the positions does not increase appreciably with more displacement. Wang et al. (2012) found a small increase in the noise level of GNSS positions under high levels of shaking, but when considering the signal-tonoise ratio of the positions, this effect is negligible
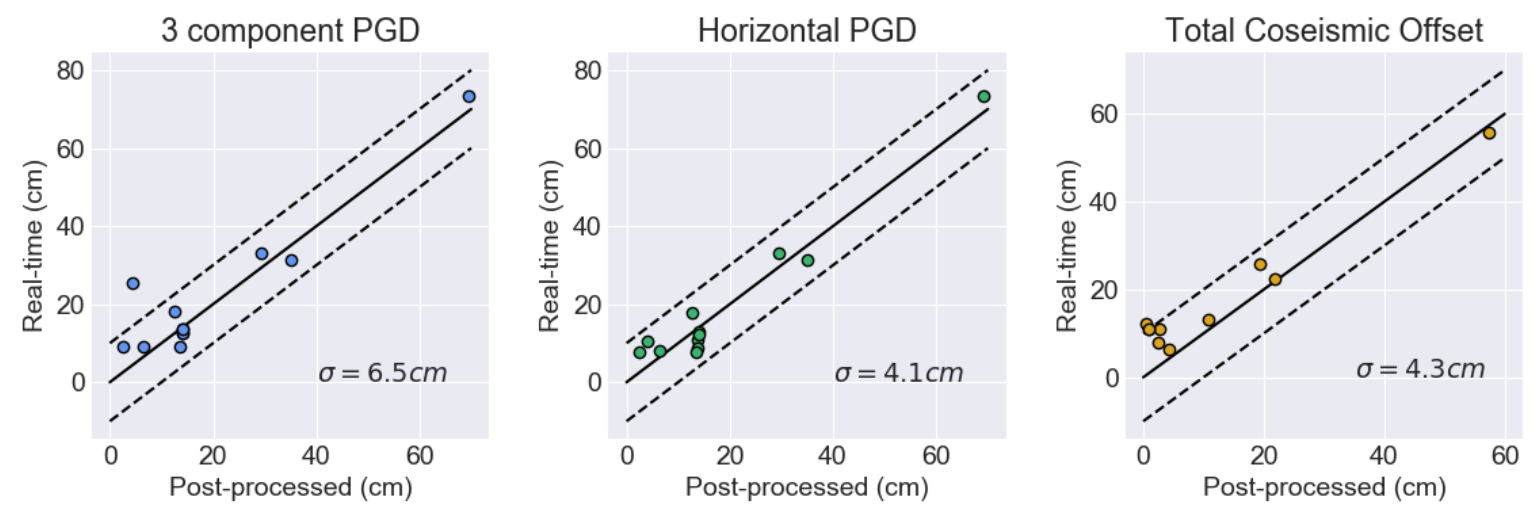

Figure 5. Comparison between real-time and post-processed PGD and coseismic offsets. The thick line indicates the 1:1 correspondence. Dashed lines indicate +/$10 \mathrm{~cm}$ from this value.

We also measure the coseismic offsets on the real-time waveforms using the moving average method used by Crowell et al. (2016) employing an S-wave travel-time mask of $2 \mathrm{~km} / \mathrm{s}$. When compared to the post-processed offsets (Figure $5 \mathrm{c}$ ) we measured a standard deviation of $4.3 \mathrm{~cm}$ in the difference between them. We also see a slight positive bias towards real-time offsets being larger than the post-processed ones. This is likely due to the long period noise identified in Figure 3.

\section{Initial Assessment of Impacts on Real-Time Performance}

Is this performance good enough for real-time seismology and rapid response? Paramount to geodetic early warning systems is the ability to properly ascertain the PGDs and coseismic offsets in a timely manner. Given the $4-6 \mathrm{~cm}$ uncertainty in PGD (Figure 5) we can quantify its impact in real-time magnitude calculations. The original PGD scaling law proposed by Crowell et al. (2013) describes the magnitude of an earthquake (M) with respect to the three-component PGD and the distance to the source $(R)$ such that

$$
\log (P G D)=A+B^{*} M+C^{*} M^{*} \log (R)
$$


Where log is the base 10 logarithm, while $A, B$, and $C$ are coefficients derived through a linear regression using past earthquakes. These coefficients have been updated several times (e.g., Melgar et al., 2015; Crowell et al., 2016; Ruhl et al., 2018,), but they are fairly consistent and lead to minimal differences in magnitude estimation. For the Ridgecrest earthquakes, we found a standard deviation of $6.5 \mathrm{~cm}$ for the three component PGD values between the real-time and postprocessed values. Using the log-summation rule, we can represent the uncertainty in the relationship as

$$
\log (P G D)+\log \left(1+/-\sigma_{P G D}\right)=A+B^{*} M+C^{*} M^{*} \log (R)
$$

Using the most recent regression coefficients from Ruhl et al. (2018), we solve for the PGD value required at certain distances to keep the total magnitude estimate variability below $+/-0.3$ magnitude units given the $6.5 \mathrm{~cm} \sigma$ value. We term this the 'critical PGD' value, this is the point to which PGD must rise in order for us to be confident that the magnitude estimation is within the specified tolerance of $+/-0.3$ magnitude units. The results are shown in Figure 6 , we find that at a distance of $100 \mathrm{~km}$, for example, the PGD value needs to be greater than $14 \mathrm{~cm}$ using the three component PGD or $9 \mathrm{~cm}$ using only horizontal components $(\sigma=4.1 \mathrm{~cm})$. Plotted in Figure 6 as well are the expected values of PGD for different magnitude earthquakes using the coefficients of Ruhl et al., (2018). We can see that for an M6 earthquake for example, stations need to be within $\sim 25 \mathrm{~km}$ of the source to exceed the critical PGD value. For an earthquake of M7, stations must be within $150 \mathrm{~km}$, and for a M8 earthquake, within $500 \mathrm{~km}$. This empirical determination of the PGD uncertainty and the critical PGD value are a useful guide to determine the effectiveness of GNSS for different sized earthquakes and at certain distances from the earthquake source. For the Ridgecrest earthquake, two thirds of the observations are above this critical PGD level, lending further confidence to the real-time positioning estimates.

One final issue we analyze briefly here is that of the sampling rate and aliasing. HRGNSS is typically collected at $1 \mathrm{~Hz}$ not because of some physical limitation of the GNSS receiver. Indeed, sample rates as high as $50 \mathrm{~Hz}$ have been reported for seismological applications (e.g. Bock et al., 2011). $1 \mathrm{~Hz}$ is preferred because GNSS is far more verbose than seismic data so faster sampling rates place undue burden on telemetry from 4+ phase and range observables per satellite per epoch (10+ satellites for multi-GNSS), as well as

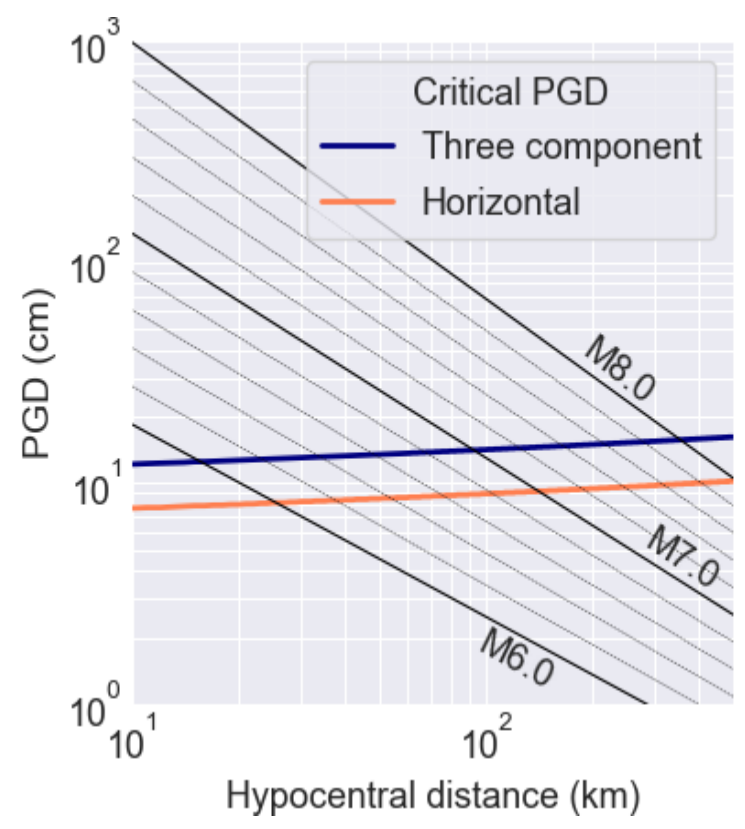

Figure 6. Critical $P G D$ value based on the threecomponent and horizontal-only values of the $P G D$ uncertainties. Also shown are the expected $P G D$ amplitude as a function of earthquake magnitude and hypocentral distance. 
extraneous receiver-dependent messages, observation corrections, and slew of other metadata. Roughly speaking, three $1 \mathrm{~Hz}$ channels of GNSS are equivalent to $6100 \mathrm{~Hz}$ channels of seismic data. As a result of this relatively slow sampling it has been posited that aliasing can lead to errors of measurement and biases in the spectral content of the recorded ground motions (e.g. Smalley, 2009), and that $10 \mathrm{~Hz}$ would be a better sampling rate for high fidelity displacements.

We do not find evidence of error introduced by the sampling rate. Figure 7 shows the 1 and $5 \mathrm{~Hz}$ total displacement waveforms and amplitude spectra for the same 3 sites as Figure 3. While there is perhaps some suggestion of aliasing from the
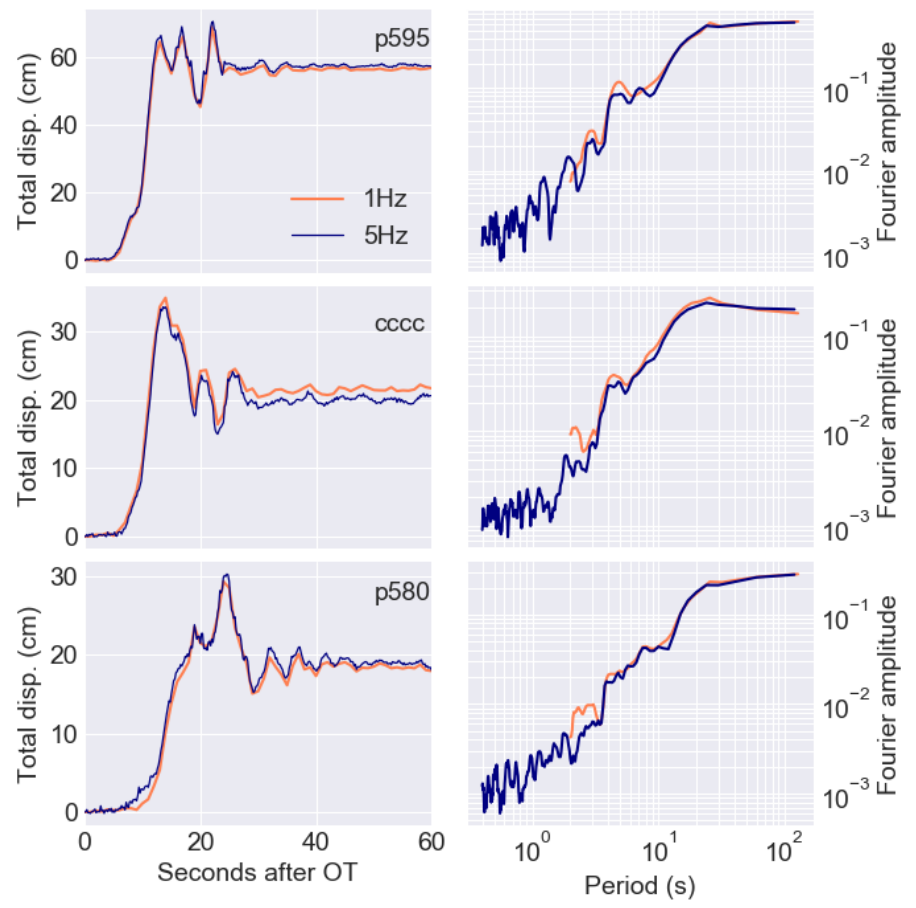

Figure 7. Comparison between $5 \mathrm{~Hz}$ and $1 \mathrm{~Hz}$ waveforms and spectra for the total displacements of the stations in Figure 2 slightly elevated spectral content between 2 and $3 \mathrm{~s}$ period in the $1 \mathrm{~Hz}$ waveforms overall the spectra are very similar to each other. Additionally, the difference in the PGD values between 1 and $5 \mathrm{~Hz}$ is quite small (Figure 8) with a standard deviation of only $0.83 \mathrm{~cm}$.

In aggregate, this shows that both of the important features of GNSS waveforms which are used for rapid source modeling, PGD and coseismic offsets, are of sufficient quality in real-time products as to be useful for warning applications. Indeed, the latency of the positions (Figure 9) measured from the field site to the position estimate being complete is of only $\sim 1-2 \mathrm{~s}$ across all the real-time sites shown in Figure 1. This latency includes the time of arrival of a position estimate minus the timestamp of the epoch itself and includes both data telemetry and processing time. For a one hour period bracketing the M7.1 earthquake the latency averaged 1.4 seconds for all stations that show resolvable coseismic offsets (Figure 9). For NOTA sites the telemetry path is from the field site to UNAVCO in Boulder, CO, and then to CWU. This extra hop introduces $\sim 250 \mathrm{~ms}$ of delay. There is a further $\sim 1 \mathrm{~s}$ delay from packetization and multiplexing of the data

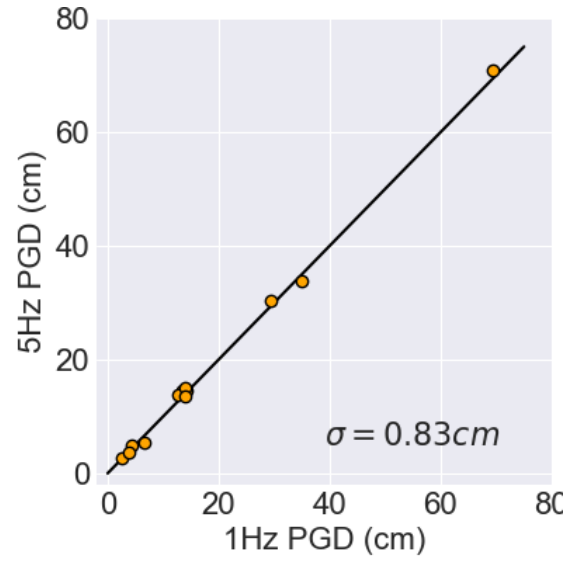

Figure 8. Difference between the $P G D$ values measured on the $1 \mathrm{~Hz}$ vs $5 \mathrm{~Hz}$ waveforms. The line indicates the 1:1 correspondence. 
to bundle all the configured sites and send them to the output socket. With PGD estimates available within $15 \mathrm{~s}$ of the origin time for the sites closest to the source of the M7.1 earthquake (Figure 2), it would have been possible for GNSS to contribute in a meaningful way to the ShakeAlert Earthquake Early Warning system during these earthquakes. Coseismic offset estimates are made soon thereafter and thus by $\sim 20$ s after the origin time (including telemetry delays) a complete preliminary characterization of the earthquake, which includes the moment tensor and a slip inversion, would be possible.

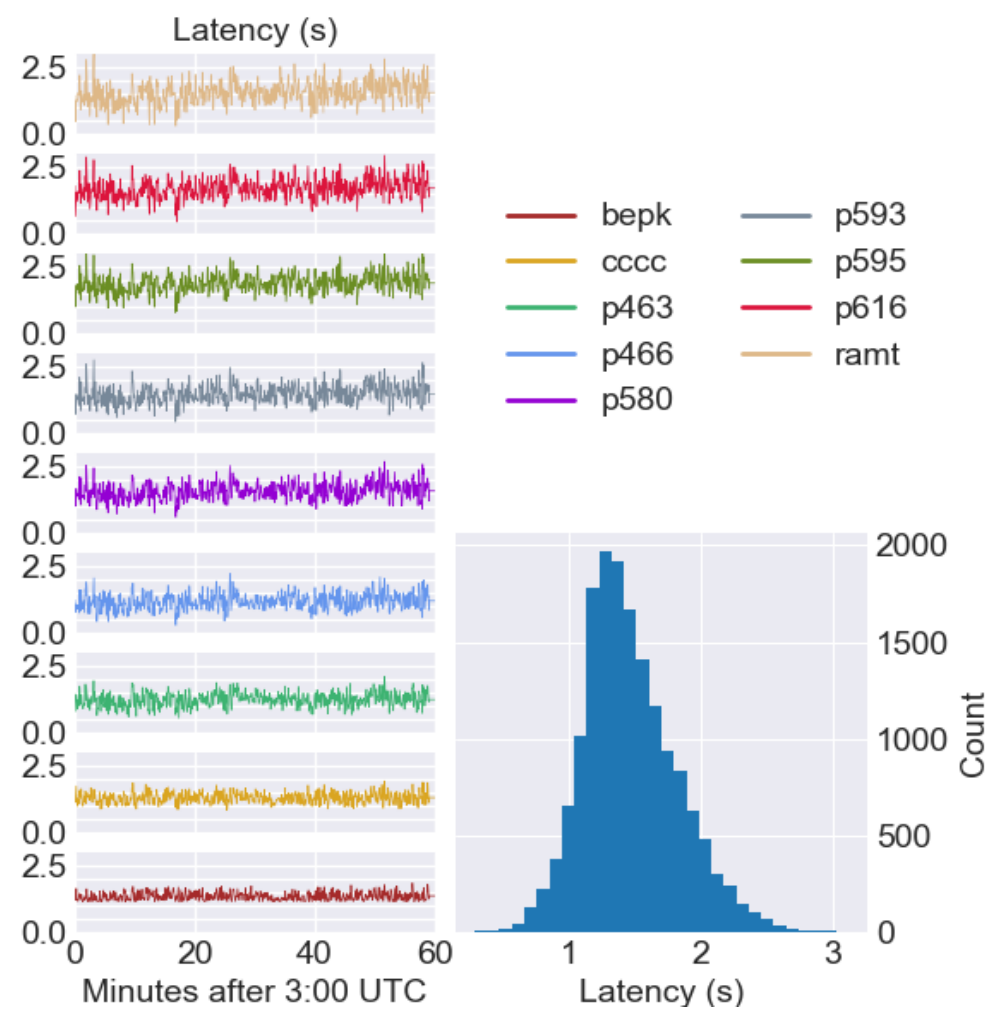

Figure 9. Positioning latency by station during a one hour period bracketing the M7.1 earthquake. Latency is defined as the time of arrival of the estimated position for a given epoch into a position database minus the timestamp of the observables themselves, and includes acquisition, telemetry, and processing time.

\section{Summary}

While RT-GNSS has been studied before and many researchers have argued that it should play a prominent role in real-time seismological systems, the 2019 Ridgecrest, CA, earthquakes are the first opportunity we have had to truly assess the end-to-end performance of an already operational RT-GNSS positioning system designed and built to monitor earthquakes. Here we find that during the events the NOTA stations operated by UNAVCO delivered data robustly and with low latency. We also find that the FastLane positioning service operated at CWU produced displacement solutions of good quality when compared to post-processed waveforms. An analysis 
of the quality of the PGD and coseismic offsets determined from the real-time data clearly shows that for moderate to large events GNSS performs well and provides useful and valuable data that can supplement traditional real-time seismological algorithms. Overall the events were an important real-world test of RT-GNSS structures for which, we argue, the system performed as expected.

\section{Data and Resources}

Real-time $1 \mathrm{~Hz}$ and post-processed 1 and $5 \mathrm{~Hz}$ three-component displacement waveforms are provided in miniSEED format and archived at Zenodo (https://zenodo.org/record/3366342). Static offsets from the GAGE soltuion can be obtained from the UNAVCO community response page https://www.unavco.org/highlights/2019/ridgecrest.html. RINEX files with the raw GNSS data can be obtained from the UNAVCO high-rate ftp archive (ftp://data-out.unavco.org/pub/highrate/) The post-processing of the GNSS waveforms was performed with the PRIDE PPP-AR code available at https://bit.ly/33Ewbey

\section{Acknowledgments}

This work was partially funded by NASA grants NNXIOAD15G and 80NSSC19K0359 and USGS-NEHRP grant G17AC00344 to Central Washington University, and RRNES18-0001 and DISASTER18-0040 to University of Oregon. Data are from Network of the Americas (NOTA) stations, funded by the National Science Foundation and operated by UNAVCO, Inc. Data based on services provided by the GAGE Facility, operated by UNAVCO, Inc. with support from the National Science Foundation and the National Aeronautics and Space Administration under NSF Cooperative Agreement EAR-1724794.

\section{References}

Beresnev, I.A. and Atkinson, G.M., (1997). Modeling finite-fault radiation from the $\omega \mathrm{n}$ spectrum. Bulletin of the Seismological Society of America, 87(1), pp.67-84.

Blewitt, G., W. C. Hammond, and C. Kreemer (2018), Harnessing the GPS data explosion for interdisciplinary science, Eos, 99, https://doi.org/10.1029/2018E0104623.

Bock, Y., Melgar, D. and Crowell, B.W., (2011). Real-time strong-motion broadband displacements from collocated GPS and accelerometers. Bulletin of the Seismological Society of America, 101(6), pp.2904-2925.

Bock, Y. and Melgar, D., (2016). Physical applications of GPS geodesy: A review. Reports on Progress in Physics, 79(10), p.106801.

Boore, D.M. and Bommer, J.J., (2005). Processing of strong-motion accelerograms: needs, options and consequences. Soil Dynamics and Earthquake Engineering, 25(2), pp.93-115.

Crowell, B.W., Bock, Y. and Melgar, D., 2012. Real-time inversion of GPS data for finite fault modeling and rapid hazard assessment. Geophysical Research Letters, 39(9).

Crowell, B.W., Melgar, D., Bock, Y., Haase, J.S. and Geng, J., (2013). Earthquake magnitude scaling using seismogeodetic data. Geophysical Research Letters, 40(23), pp.6089-6094. 
Crowell, B.W., Schmidt, D.A., Bodin, P., Vidale, J.E., Gomberg, J., Renate Hartog, J., Kress, V.C., Melbourne, T.I., Santillan, M., Minson, S.E. and Jamison, D.G., (2016). Demonstration of the Cascadia G-FAST geodetic earthquake early warning system for the Nisqually, Washington, earthquake. Seismological Research Letters, 87(4), pp.930-943.

Geng, J., Bock, Y., Melgar, D., Crowell, B.W. and Haase, J.S., (2013). A new seismogeodetic approach applied to GPS and accelerometer observations of the 2012 Brawley seismic swarm: Implications for earthquake early warning. Geochemistry, Geophysics, Geosystems, 14(7), pp.2124-2142.

Geng, J., Chen, X., Pan, Y., Mao, S., Li, C., Zhou, J. and Zhang, K., (2019). PRIDE PPP-AR: an open-source software for GPS PPP ambiguity resolution. GPS Solutions, 23(4), p.91.

Herring, T.A., Melbourne, T.I., Murray, M.H., Floyd, M.A., Szeliga, W.M., King, R.W., Phillips, D.A., Puskas, C.M., Santillan, M. and Wang, L., (2016). Plate Boundary Observatory and related networks: GPS data analysis methods and geodetic products. Reviews of Geophysics, 54(4), pp.759-808.

Kawamoto, S., Ohta, Y., Hiyama, Y., Todoriki, M., Nishimura, T., Furuya, T., Sato, Y., Yahagi, T. and Miyagawa, K., (2017). REGARD: A new GNSS-based real-time finite fault modeling system for GEONET. Journal of Geophysical Research: Solid Earth, 122(2), pp.1324-1349.

Larson, K.M., Bodin, P. and Gomberg, J., (2003). Using 1-Hz GPS data to measure deformations caused by the Denali fault earthquake. Science, 300(5624), pp.1421-1424.

Larson, K.M., (2019). Unanticipated Uses of the Global Positioning System. Annual Review of Earth and Planetary Sciences.

Melbourne, T.I, Stough, T., Bar-Sever, Y., Bock, Y., Song, T. (2018), Operationalizing GNSS as a Fourth Observation for Local Tsunami Warning, Abstract NH34B-02 presented at 2018 Fall Meeting, AGU, Washington, D.C., 10-14 Dec.

Melgar, D., Bock, Y. and Crowell, B.W., (2012). Real-time centroid moment tensor determination for large earthquakes from local and regional displacement records. Geophysical Journal International, 188(2), pp.703-718.

Melgar, D., Bock, Y., Sanchez, D. and Crowell, B.W., (2013). On robust and reliable automated baseline corrections for strong motion seismology. Journal of Geophysical Research: Solid Earth, 118(3), pp.1177-1187.

Melgar, D., Crowell, B.W., Geng, J., Allen, R.M., Bock, Y., Riquelme, S., Hill, E.M., Protti, M. and Ganas, A., (2015). Earthquake magnitude calculation without saturation from the scaling of peak ground displacement. Geophysical Research Letters, 42(13), pp.5197-5205.

Murray, J.R., Crowell, B.W., Grapenthin, R., Hodgkinson, K., Langbein, J.O., Melbourne, T., Melgar, D., Minson, S.E. and Schmidt, D.A., (2018). Development of a geodetic component for the US West Coast earthquake early warning system. Seismological Research Letters, 89(6), pp.2322-2336.

Nikolaidis, R. M., Bock, Y., de Jonge, P. J., Shearer, P., Agnew, D. C., and Van Domselaar, M. ( 2001),Seismic wave observations with the Global Positioning System, J. Geophys. Res., 106, 21,897-21,916.

O'Toole, T.B., Valentine, A.P. and Woodhouse, J.H., (2012). Centroid-moment tensor inversions using high-rate GPS waveforms. Geophysical Journal International, 191(1), pp.257-270.

Prieto, G.A., Parker, R.L. and Vernon III, F.L. (2009). A Fortran 90 library for multitaper spectrum analysis. Computers \& Geosciences, 35(8), pp.1701-1710. 
Riquelme, S., Bravo, F., Melgar, D., Benavente, R., Geng, J., Barrientos, S. and Campos, J., (2016). W phase source inversion using high-rate regional GPS data for large earthquakes. Geophysical Research Letters, 43(7), pp.3178-3185.

Ruhl, C.J., Melgar, D., Geng, J., Goldberg, D.E., Crowell, B.W., Allen, R.M., Bock, Y., et al., (2018). A global database of strong-motion displacement GNSS recordings and an example application to PGD scaling. Seismological Research Letters, 90(1), pp.271-279.

Smalley Jr, R., (2009). High-rate GPS: How High do we need to go?. Seismological Research Letters, 80(6), pp.1054-1061.

Wang, G., F. Blume, C. Meertens, P. Ibanez, and M. Schulze (2012), Performance of high-rate kinematic GPS during strong shaking: Observations from shake table tests and the 2010 Chile earthquake, J. Geodetic Science, 2(1), 15-30, doi: 10.2478/v10156-011-0020-0.

Wright, T.J., Houlié, N., Hildyard, M. and Iwabuchi, T., (2012). Real-time, reliable magnitudes for large earthquakes from $1 \mathrm{~Hz}$ GPS precise point positioning: The 2011 Tohoku-Oki (Japan) earthquake. Geophysical research letters, 39(12).

Zumberge, J.F., Heflin, M.B., Jefferson, D.C., Watkins, M.M. and Webb, F.H., (1997). Precise point positioning for the efficient and robust analysis of GPS data from large networks. Journal of geophysical research: solid earth, 102(B3), pp.5005-5017. 